\title{
Instrumental Demonstration of Hypnotic Flaccid Paralysis of Corrugator Muscles and Its Use in Esthetic Medicine
}

\author{
Edoardo Casiglia ${ }^{1,2}$, Liliana Mentesana² \\ ${ }^{1}$ Department of Medicine, University of Padua, Italy \\ ${ }^{2}$ Institute Franco Granone, Italian Centre of Clinical and Experimental Hypnosis, Turin, Italy
}

\begin{abstract}
Flaccid paralysis is very easy to produce in hypnosis and is often used in didactic setting. This paper is aimed at demonstrating that hypnotic flaccid paralysis is a real, measurable and reproducible phenomenon that belongs to the Galilean science and can be studied with the methods of human physiology.

Ten participants ( 5 highly hypnotizable subjects and 5 control patients highly responsive to botulinum toxin) were recruited. Through a non-invasive electromyograph, muscular tension was previously measured (in $\mu \mathrm{V}$ ) in all participants at the corrugator muscles of the forehead. The 5 highs then underwent in experimental setting hypnotic induction followed by the suggestion of flaccid paralysis of the corrugators. The 5 control patients received injection of a paralytic dose of botulinum toxin at the level of the corrugators. Both groups were visually observed, and electromyography was repeated while the participant received the commands «corrugate» or «don't corrugate».

Both participants undergoing hypnosis and patients receiving the toxin were patently unable to corrugate. Furthermore, the muscular tension recorded at the corrugator was even lower in the former than in the latter, probably due to the additional relaxing effect of hypnosis. The results demonstrate that hypnotic flaccid muscular paralysis is real, and open a way to use hypnotic commands in preventing unconscious corrugation that leads to irreversible dermal fractures of foreheads. In this indication, hypnosis could be more effective (and of course less invasive) that injection of botulinum toxin. Long-lasting studies are mandatory to confirm the present results recorded in acute experimental setting.

Keywords: Face, dermal, fracture, winkles, mimics, physiology, human
\end{abstract}

\section{INTRODUCTION}

Bachground. In the last years the Laboratory of Experimental Hypnosis of the Department of Medicine of the University of Padua in collaboration with the Institute Franco Granone of Turin (Italy) has demonstrated that,

*Correspondence: edoardo.casiglia@unipd.it

Prof. Edoardo Casiglia, Department of Medicine University of Padua

Via Giustiniani No. 2, 35128 Padua, Italy

Tel +39-049-8212277, Fax +39-049-8754179

\section{Sleep and Hypnosis}

Journal homepage:

www.sleepandhypnosis.org

ISSN:1302-1192 (Print) 2458-9101 (Online) through suggestions given to a subject in deep hypnosis, it is possible to induce cognitive modifications which can be studied in controlled experimental setting. Thus, it has been shown that the effects of hypnotic suggestions are not merely subjective but induce objective cerebral and physiological modifications that are real in everyday physical world.

\section{Hypnosis in esthetic medicine, a new field of} research. Esthetic medicine is a multidisciplinary branch of medicine dealing with wellbeing of health people. World Health Organization states that health is not absence of disease, but rather a complete state of 
physical, psychic and social wellbeing. When the needs related to survival are satisfied, other needs intervene involving the emotional and relational sphere and contributing to improvement of quality of life. To take care of physical aspect therefore becomes per se a need and in many cases a priority. Eliminating an unpleasant blemish, even if non detrimental for health, appearing younger, correcting something we don't like, positively modifies mood and the mental behavior with positive chain reactions in different aspects of personal life. It is not incorrect to state that esthetic medicine is the medicine of wellbeing.

In this view, botulinum toxin is one of the most requested therapies and the most gratifying. Many studies show that inhibition of contractility of the corrugator muscles expressing rage or sadness has a positive effect on depressed patients. Hexsel et al. (2013) evaluated selfesteem and depressive symptoms in 25 subjects with and in 25 without depression, and treated both groups with botulinum toxin injected in the corrugators. They observed a significant improvement of all the studied parameters. Hennenlotter et al. (2009) demonstrated that subjects in which the contraction of corrugator muscles (and then the possibility to express rage) was blocked, functional magnetic resonance showed a reduced activity at the level of the amigdala and of the limbic system, two structures involved in emotional activity. It can be postulate that modifications of expressive dynamics have cerebral functional reverberations.

Other effect to be taken into account are facial expression and emotional contagion. Botulinum treatment, acting on mimic muscles involving expression with negative traits such as rage, sadness, worry, anxiety and fear are important for the relationship with other people, as the transmission - even unconscious - of a negative sentiment don't put the interlocutor in a condition of serenity towards us. Charles Darwin attributed to the corrugator muscles a great impact to the expression of emotions, that he considered very important for man's evolution. He dedicated a volume to the observation of emotion in man and animal, with intense facial expression illustrating the book that were a great novelty for that time.
Botulinum toxin. Botulinum toxin was used for the first time in medicine in 1970 for treatment of squinting, and then for spasticity, blepharospasm, hyperhidrosis, neurologic urinary bladder, esophageal achalasia and headache. It was at the end of the ' 80 s of XX Century that the dermatologist Alastair Carruthers and the ocular surgeon Jean Carruthers observed an esthetic positive effect after injection of the toxin in facial wrinkles. This happened by change: in the frame of a therapy for blepharospasm, after observing the success of the treatment for the glabellar wrinkles, the two examined in depth the use of this tecnique for the off label indication of correcting not only glabellar winkles but also the other hyper-functional facial wrinkles (Carruthers and Carruthers, 1992, 1998; Carruthers, 2002).

An important muscle. The corrugator supercilii is a flat and thin muscle, arcuate like the eyebrow to which it belongs. It is placed in a deeper plane in comparison to orbicular and frontal, from which it is covered. It originates from the frontal bone in the more medial part of the supraorbital ridge, proceeds upwards and laterally and, at the level of the supraorbital notch, breaks down in bundles that intertwine with those of the orbicularis oculi and of the frontal muscle and ends in the supracilial skin. This muscle holds the eyebrow causing longitudinal skinfolds laterally to the nasal root. It concurs with the orbicular and the procerus in defending eyes from a too vivid light or from foreign bodies and contracts during accommodation. As a mimic muscle, it takes action with other muscles to express concentration or in occasion of muscular efforts. Due to contraction of this muscle, physiognomy assumes an expression of suffering, pain or concentration.

Wrinkles. Wrinkles are commonly classified in four groups: 1) no wrinkles either in statics or in dynamics; 2) wrinkles with movement only; 3 ) wrinkles also at rest; 4) profound wrinkles with dermal fractures.

A dermal fracture happens after repeated and prolonged muscular contractions. This leads to degradation of the elastic fibers and of collagen, and to dermal thinning and atrophy. This fracture persists also 
when skin is stretched with fingers.

Botulinum toxin prevents dermal fractures by preventing contraction of the corrugator. Preventing this sequence of events by means of hypnotic commands, eventually reiterated, could be equivalent to a hypnotic lifting, i. e. to a non-pharmacological anti-ageing therapy.

Aim of the present study was to induce by a hypnotic command a botulinum-like effect of flaccid paralysis of the corrugators and to compare it to the paralysis induced by botulinum toxin. Apart from the speculative meaning of the research, that would furnish the instrumental confirmation of reality and measurability of flaccid hypnotic paralysis, another advantage was to propose an alternative to those patients that, although disturbed by the «thinker wrinkles», don't want to block facial mimics with a drug.

\section{METHODS}

Study groups. Two groups of people of 5 subjects ( 1 men and 4 women) each were enrolled. One group (mean age $36 \pm 8$ years, range 24 to 43 ) underwent the hypnotic procedure specified below, the other one (mean age $41 \pm 9$, range 48 to 60 ) underwent administration of botulimun toxin injection with the technique described below. The subjects of the group «hypnosis» were highs who already knew hypnosis and the author E.C., those of the group «botulinum toxin» were patients who already knew botulinum toxin and the author L.M. All gave informed consent, and the institutional ethics committee specifically authorized hypnotism.

Group «hypnosis». In all subjects of the group «hypnosis» 4 measurements were performed, i. e. two in pre-hypnotic basal conditions («don't corrugate» vs. «corrugate») and two in hypnotic flaccid paralysis («don't corrugate» vs. «corrugate»). Before the experiment, each subjects individually received an instruction course about approximate anatomy and function of corrugator muscles. With a picture, their position on the forehead was also visualized, and the points of real or hallucinated toxin injection were indicated.
It was explained in terms good for profane that botulinum toxin acts by inhibiting electric conduction from neural axon to muscles, so blocking the ability of corrugating.

During hypnotic suggestion, it was proposed to imagine that touching the corrugators were equivalent to injection of an active dose of botulinum toxin, and that following this procedure the forehead would be smoothed and subjects would not be able to corrugate on demand. This effect would disappear at the end of the setting.

We consider hypnosis as a tool (Casiglia et al., 1994, 2012), and our research group previously demonstrated that hypnotic and post-hypnotic commands are able to act in physical world, increasing physical performance (Tikhonoff et al., 2012), dilating arterial and venous vessels (Casiglia et al., 2015a), producing local analgesia (Casiglia et al., 2007, 2015, 2016; Facco et al., 2011, 2013) and general anesthesia (Casiglia et al., 2015) by blocking both non-trigeminal and trigeminal algic stimuli before they arrive to the brain sensitive areas, and reproduce the physical effects of hemorrhage (Casiglia et al., 1997), and more in general have effects that are measurable in a scientific laboratory with the methods of human physiology (Casiglia et al., 2012). We were therefore confident that the command «you are unable to corrugate» would be obeyed. It remained to clarify its degree of efficacy in reducing the attitude to corrugate.

Group «botulinum toxin». In this group, 4 measurements were equally performed, two before injection («don't corrugate» vs. «corrugate») and two 1 week after injection («don't corrugate» vs. «corrugate»), as usual in esthetic medicine to give active principle the time necessary to block the release of acetylcholine from neuro-muscular plaques (Vickers et al., 2001). Toxin allowed by the Italian Ministry of Health for esthetic medicine was used, diluted with $0.63 \mathrm{ml}$ saline solution to obtain a transparent solution containing 125 Speywood units of active principle at a concentration of 10 units in $0.05 \mathrm{ml}$ solution. Four injections of 10 units each were performed (two for each muscle). As the effect of toxin is 
dose-dependent, patients needing this dose to obtain smoothing of corrugators were selected ${ }^{\ddagger}$. Botulinum toxin was injected in sterility in the context of each corrugator at the dose of 20 unit per muscle in two separate loci using a 1-ml syringe graduated in botulinum units with a $32 \mathrm{G}$ needle (Figure 1).

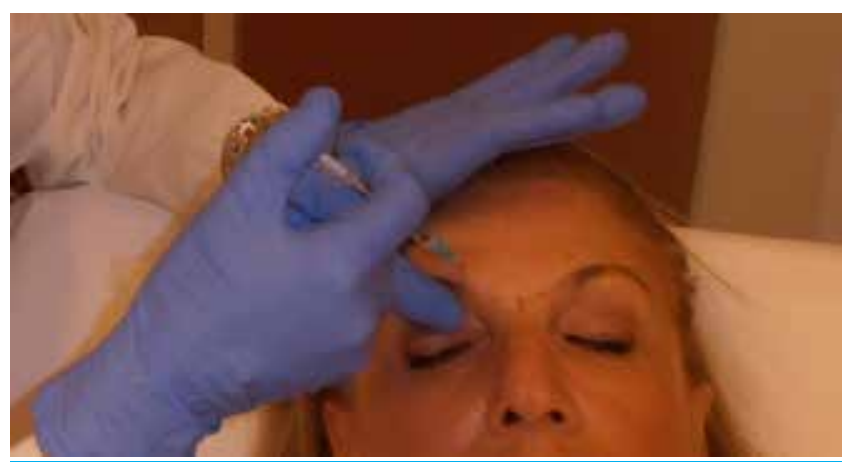

Figure 1: Injection of botulinum toxin into the corrugator in subject No. 2.

The botulinum toxin presents seven variants. That used in medicine is the product of the obligate anaerobes Clostridium botulinum type $A$, the less aggressive. This variant is safe, no risk of systemic diffusion exists, and the doses used are very far from the toxic ones. The botulinum toxin is a $100 \mathrm{KDa}$ double-chain polypeptide linked through disulfide bridges to a $50 \mathrm{KDa}$ chain. The heavy chain is important for endocytosic penetration in the axonal extremity, while the light one is a protease attacking some proteins of neuromuscular junctions, including SNAP 25. The result of this block is lack of release of acetylcholine from presynaptic vesicles and consequently lack of muscular contraction. Muscular paralysis is reversible thanks to the axonal sprouting, representing the reparative reaction of the plaque to block: an accessory axon is made, so that the primary plaque is restored to complete functionality.

Instrumental measurements. For the measurement of muscular contraction of the corrugator, a non-invasive surface electromyography was used (MicroEMG, OT
Bioelettronica, Turin, Italy) developed by Dario Farina (UMG, Goettingen University, Germany), a single-canal device employing a special concentric bipolar single electrode, with a gain of $980 \mathrm{~V} / \mathrm{V}$, a band width of 10 to $500 \mathrm{~Hz}$ and a sampling frequency of $1 \mathrm{kHz}$. The device saves a data file when the operator presses in sequence the button «start» and the button «stop». Each recording had to last about 20 seconds, then the tracing (see below) was processed in order to obtain a graph. From each graph, the peak value and the nadir of contractions, to be used in statistical analysis, were extrapolated.

\section{RESULTS}

All subjects of the group «hypnosis» stated after dehypnotization to have experimented a nice and profound trance and did not complain of any side effect. Three of then did not remember the procedure having developed spontaneous amnesia, while two were able to describe in details the experimental procedure.

The hypnotic flaccid paralysis is shown in Figure 2, were the smoothing of the corrugator with impossibility to corrugate when the hypnotic command was operative is shown.
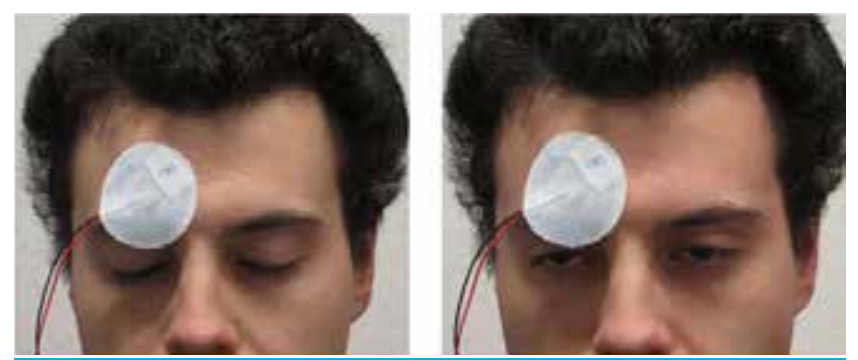

Figure 2. Effect of the instruction "corrugate» in pre-hypnotic basal conditions and in hypnotic flaccid paralysis in subjects No. 8.

The chemical flaccid paralysis induced by the botulinum toxin is shown in Figure 3, where the muscular smoothing after injection is clearly shown.

Figure 4 shows the supplement of contraction of

\#In the same setting, to complete the esthetic procedure, 10 units of toxin were also injected in the procerus, that is not involved in this research. 

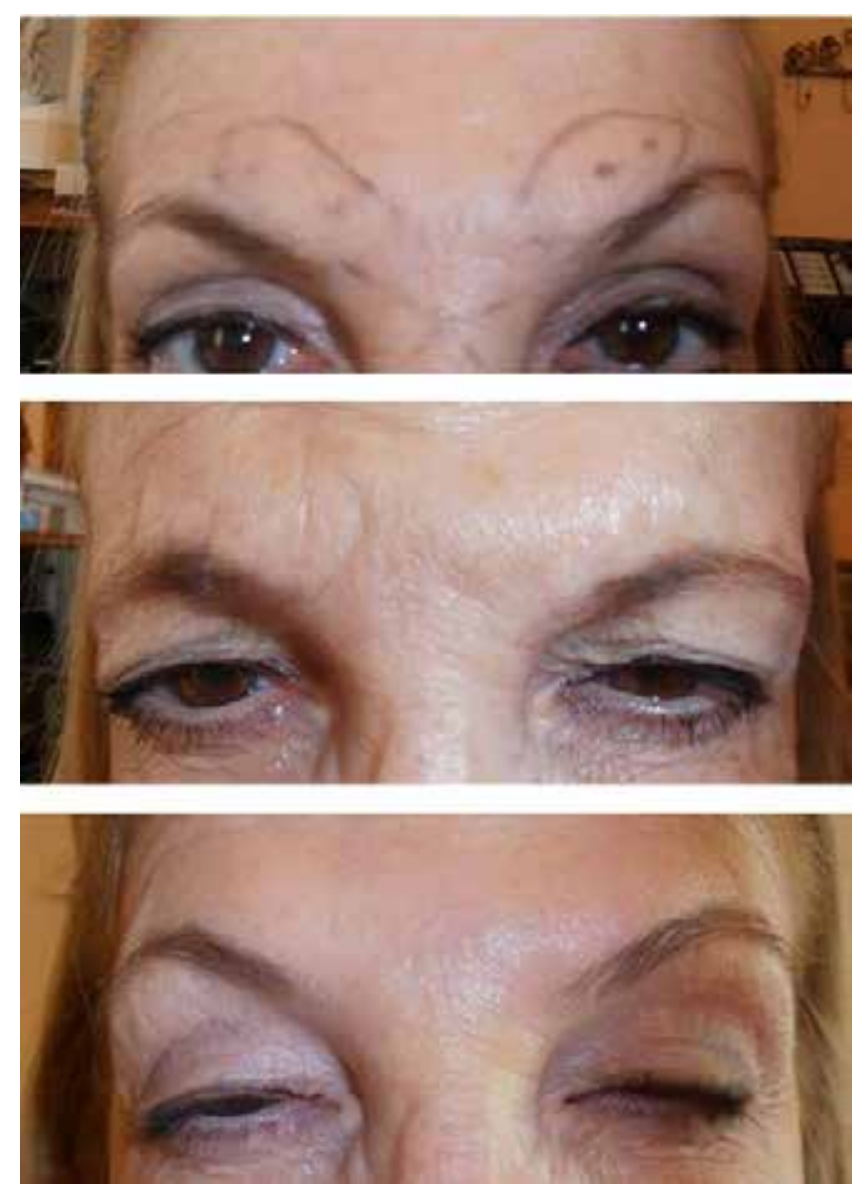

Figure 3. Effect of the instruction "corrugate» before toxin injection and in flaccid paralysis due to botulinum toxin in subject No. 2. Upper panel: basal condition before injection, «don't corrugate». Internediate panel: basal condition before injection, «corrugate». Lowen panel: after injection, «corrugate».

corrugator muscles due to the instruction «corrugate» in the two groups.

In subjects who had received the botulinum toxin, this excess (quantifiable in $36.5 \mu \mathrm{V}$ ), is the residual ability to contract after the toxin acted blocking the neuro-muscular junction, representing the basis for the evaluation of the hypnotic effect.

In subjects receiving the hypnotic command to be paralytic, there was no excess contraction, and the electromyographic tracings recorded during corrugation were lower than those recorded in pre-hypnotic conditions (Figure 5). Not only this, but the instruction «corrugate» produced a deficit of contraction $(-5.6 \mu \mathrm{V})$ due to relaxation accompanying the hypnotic procedure.

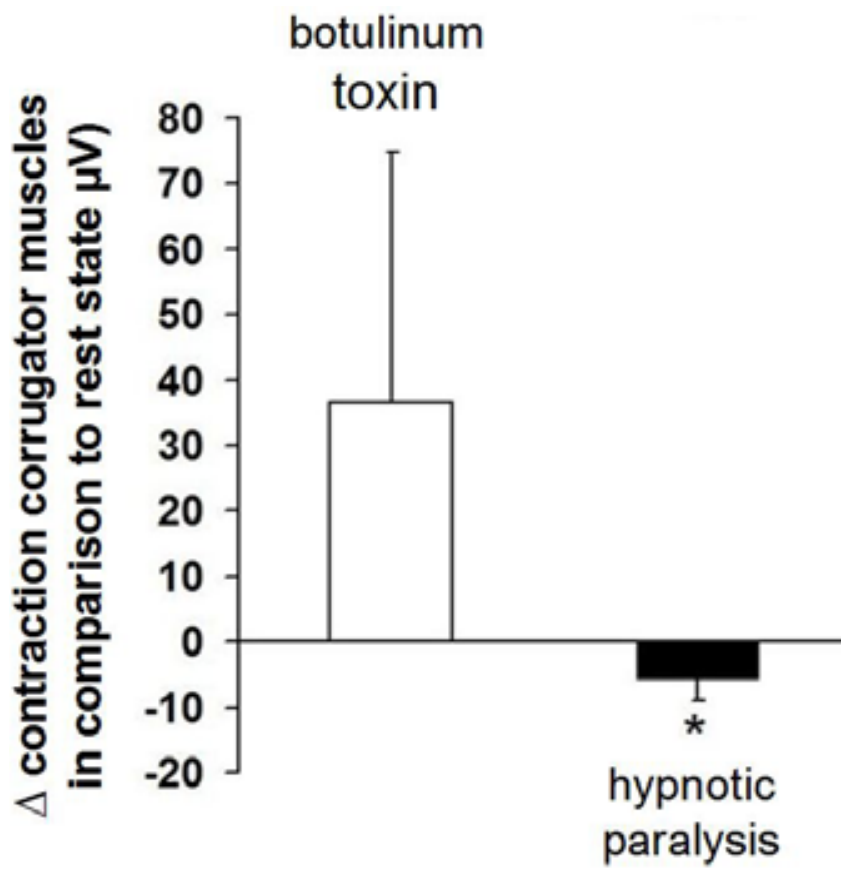

Figure 4. Supplement of contraction of corrugators after the instruction "corrugate» in the 5 subjects receiving botulinum toxin ( $\square$ ) and in the 5 receiving the hypnotic command of flaccid paralysis ( $\square$ ). The two degrees of paralysis are compared ( ${ }^{*} p<0.01$ vs. botulinum toxin).

\section{DISCUSSION}

The results of the study presented herein show that a treatment with botulinum toxin at canonical doses is efficacy in reducing contractility of corrugator muscles, leaving as residual contractile effect nothing more than $36.5 \mu \mathrm{V}$ muscular electric tension. This is not surprising for doctors who use this toxin in everyday practice.

What it is surprising is the paralytic effect of the hypnotic command of neuro-muscular block. This effect is even greater than that of the toxin, with a difference in favor of the former of $42 \mu \mathrm{V}$, biologically evident and statistically significant.

The hypnotic command of flaccid paralysis, often used in didactic setting where astonishes novices, therefore qualifies itself as a paralytic method absolutely real, scientifically measurable and superior to the chemical denervation deriving from a pharmacological treatment consolidated in clinical setting.

The visual analysis of mimics shown in Figure 2 for 


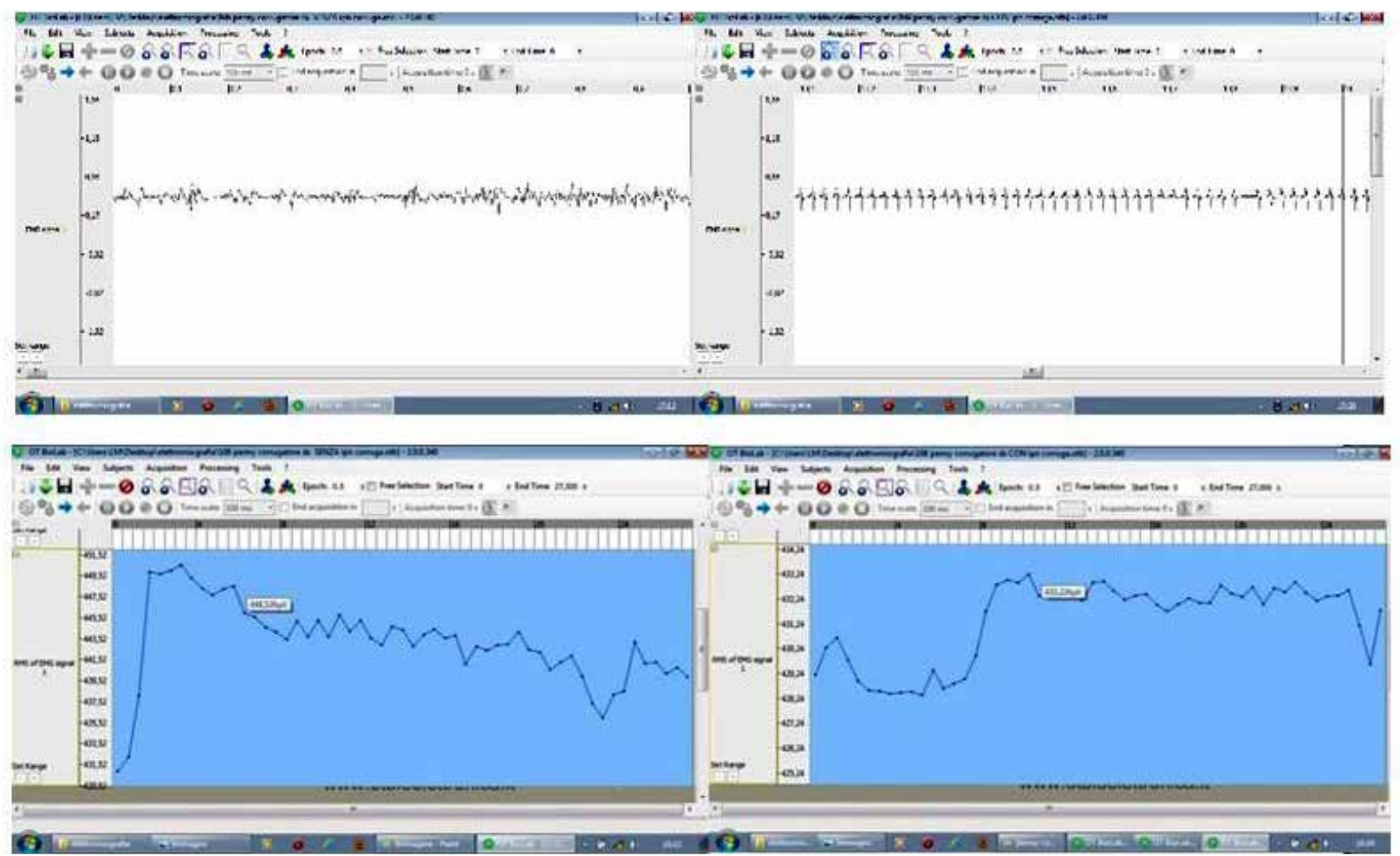

Figure 5: Electromyography during corrugation. When the hypnotic command of flaccid paralysis is active, graph values are constantly lower and more leptokurtic than those recorded in pre-hypnotic basal conditions $(444.4 \pm 1.7$ $\mu \mathrm{V}, 95 \% \mathrm{Cl} 443.8-445.0$, vs. $432.5 \pm 0.4 \mu \mathrm{V}, 95 \% \mathrm{Cl} 432.3-432.6, \mathrm{p}<0.01)$. Upper panels show the tracing in extenso, lower panels in the middle period. Left panels shows pre-hypnotic basal conditions, right panels hypnotic command of paralysis.

hypnosis and in Figure 3 for botulinum toxin also put in evidence a greater effect of basal relaxation, before the motor command is requested. This effect is not present in subjects who are ready to receive the toxin and present in those ready to receive the hypnotic paralytic command, qualifying hypnosis as an excellent method of sedation in people getting close to an intervention of esthetic medicine, an affect that is lacking in common clinical setting.

It is not worthy that, in esthetic medicine, botulinum toxin is administered not to remove the expression wrinkles but to prevent diurnal and particular night-time corrugation that in the long term will lead to dermal fractures disfiguring permanently the face, giving to it a constant expression of worry and depression. As recently elucidated (Wolmer et al., 2014), the facial expression is translated into an inner feeling with cerebral modifications that during time leads really to worry and depression. In this view, treatment with botulinum toxin represents a prevention in a psychodynamic sense too. Behavioral therapy suggests to maintain a relaxed expression, even unmotivated, in order to feel really relaxed. Furthermore, an expression with furrowed brows, corresponding or not to an inner manner, predisposes the interlocutor to negative responses, altering social relationships.

Hypnosis, with its even unexpectedly strong paralyzing attitude, is fit to substitute with full wrights the botulinum treatment. Of course, the results of the present research are limited to the experimental context of about 1 hour and do not guarantee that the effect will be long-lasting as that of the pharmacological preparation. Other longlasting studies are mandatory to clarify if hypnosis is able to substitute completely the botulinum toxin.

Conflicts of interest. The authors do not declare any conflict of interest. 


\section{References}

Carruthers A., Carruthers, J. (1998). History of the cosmetic use of botulinum: A exotoxin. Dermatological Surgery, 24, 1168-1170.

Carruthers, A. (2002). Botulinum toxin type A: history and current cosmetic use in the upper face. Disease-a-Month, 48, 299-322

Carruthers, A., Carruthers, J. (1992). Treatment of glabellar frown lines with botulinum A exotoxin. The Journal of Dermatologic Surgery and Oncology, 18, 17-21

Casiglia, E., Mazza, A., Ginocchio, G., Onesto, C., Pessina, A.C., Rossi, A.M., Cavatton, G., Marotti, A. (1997). Haemodynamics following real and hypnosis-simulated phlebotomy. American Journal Clinical Hypnosis, 4, 368-375.

Casiglia, E., Rempelou, P., Tikhonoff, V., Giacomello, M., Finatti, F., Albertini, F., Favaro, J, Lapenta, A.M., Facco, E. (2016). Hypnotic focused analgesia obtained through body dysmorphism prevents both pain and its cardiovascular effects. Sleep and Hypnosis, in press.

Casiglia, E., Rempelou, P., Tikhonoff, V., Guidotti, F., Pergher, V., Giordano, N., Testoni, I., Facco, E. (2015). Hypnotic general anesthesia vs. focused analgesia in preventing pain and its cardiovascular effects. ATINER's Conference Paper Series ISSN 2241-2891.

Casiglia, E., Rossi, A.M., Mazza, A., Cavattoni, G., Colangeli, G., Ginocchio, G., Di Menza, G., Marotti, A., Onesto, C., Pegoraro, L., Pessina, A.C. (1994). Hypnosis as a tool for evaluating the cortical component of haemodynamic variations. pilot study during blood letting. High Blood Pressure, 3 (Suppl.3), 8.

Casiglia, E., Schiavon, L:, Tikhonoff, V., Haxhi Nasto H., Azzi, M., Rempelou, P., Giacomello, M., Bolzon, M., Bascelli, A., Scarpa, R., Lapenta, A.M., Rossi, A.M (2007). Hypnosis prevents the cardiovascular response to cold pressor test. American Journal Clinical Hypnosis, 49, 255-266.

Casiglia, E., Tikhonoff, V., Rossi, A.M (2015). Positive hallucinations. In: Treatise of hypnosis and other modifications of consciousness. CLEUP, Padova (pages 593-601).
Casiglia. E., Tikhonoff, V., Giordano, N., Regaldo, G., Tosello, M.T., Rossi, A.M., Bordin, D., Giacomello, M., Facco, E. (2012). Measured outcomes with hypnosis as an experimental tool in a cardiovascular physiology laboratory. International Journal of Clinical and Experimental Hypnosis, 60, 241-261.

Facco, E., Casiglia E., Masiero, S., Tikhonoff, V., Giacomello, M., Zanette, G. (2011). Effects of hypnotic focused analgesia on dental pain threshold. International Journal of Clinical and Experimental Hypnosis, 59, 454-468.

Facco, E., Pasquali, S., Zanette, G., Casiglia, E. (2013). Hypnosis as sole anaesthesia for skin tumour removal in a patient with multiple chemical sensitivity. Anaesthesia, 68, 961-965.

Hennenlotter, A., Dresel, C., Castrop, F., Ceballos-Baumann, A.O., Wohlschläger, A.M., Haslinger B. (2009). The link between facial feedback and neural activity within central circuitries of emotion. New insights from botulinum toxin - induced denervation of frown muscles. Cerebral Cortex, 19, 537-542.

Hexsel, D., Brum, C., Siega, C., Schilling-Souza, J., Dal Forno, T., Heckmann, M., Rodrigues, T.C. (2013). Evaluation of self-esteem and depression symptoms in depressed and nondepressed subjects treated with onabotulinumtoxin A for glabellar lines. Dermatological Surgery, 39, 1088-1096.

Tikhonoff, V., Azzi, D., Boschetti, G., Giordano, N., Rempelou, P., Giacomello, M., Tosello, M.T., Facco, E:, Rossi, A.M., Lapenta, A.M., Regaldo, G., Palatini, P., Casiglia, E. (2012). Increase of isometric performance via hypnotic suggestion: experimental study over 10 young healthy volunteers. Contemporary Hypnosis and Integrative Therapy, 29, 352-262.

Wollmer, M.A., Kalak, N., Jung, S., de Boer, C., Magid M, Reichenberg. J.S., Brand, S., Holsboer-Trachsler, E., Kruger, T.H. (2014). Agitation predicts response of depression to botulinum toxin treatment in a randomized controlled trial. Frontiers in Psychiatry, 5, 1-5. 